\title{
Central Nervous System Necrosis, CTCAE
}

National Cancer Institute

\section{Source}

National Cancer Institute. Central Nervous System Necrosis, CT CAE. NCI Thesaurus.

Code C143360.

A disorder characterized by a necrotic process occurring in the brain and/or spinal cord. 